\title{
Clinical Value of Renal Biopsy in Patients with IgA Nephropathy
}

\author{
Qi Jiang, Yingying Liu* \\ Department of Nephrology, China-Japan Union Hospital of Jilin University, Changchun, Jilin, 130033 \\ ${ }^{*}$ Corresponding author
}

Keywords: Clinical Value, Renal Biopsy, IgA Nephropaty.

\begin{abstract}
This paper describes the renal pathology of patients with IgA nephropathy in the presence of chronic renal insufficiency and its clinical relevance. The clinical and pathological data of 106 patients with IgA nephropathy complicated with chronic renal insufficiency were divided into three groups according to the blood muscle and muscle. The pathological changes of kidney were graded according to Lee classification criteria and Hass classification criteria respectively. Katafuhci half Quantitative integral method is used to determine glomerular, tubulointerstitial and vascular lesions. To study the correlation between Scr, CCr, other clinical indicators and renal pathology, multiple regression analysis was used to analyze the factors influencing the level of blood-muscle drinkers.
\end{abstract}

\section{Introduction}

IgA nephropathy is a common glomerular disease in the world. Due to limitations of renal biopsy indications, renal biopsy is often abandoned clinically when patients with IgA nephropathy progress to chronic renal insufficiency, can not use the best treatment. To date, no pathological studies have been reported at home and abroad on patients with IgA nephropathy in chronic renal insufficiency. Large sample reports of renal biopsy in patients with IgA nephropathy clinically associated with chronic renal insufficiency under cautious operation and close observation, Observing the pathological characteristics of patients and their relationship with the clinic for clinicians to determine the degree of renal disease based on clinical features and renal function in patients with IgA nephropathy to provide a basis for treatment options [1].

\section{Materials and Methods}

Forty-one hundred and twelve patients with clinically small proteinuria diagnosed as IgA N by renal biopsy in our hospital from January 2005 to January 2010 were selected. IgA N is based on IgA deposition in the immunofluorescent mesangial area and excludes renal impairment such as systemic lupus erythematosus, Henoch-Schonlein purpura, chronic liver disease and kidney transplantation. All patients with systemic lupus erythematosus serological tests were negative. According to renal function is divided into normal renal function group and renal dysfunction group. A small amount of proteinuria in this study was defined as a quantitative urinary protein $<1 \mathrm{~g}$ for 24 hours (at least two tests repeated). Renal dysfunction was defined as serum creatinine $>110 \mu \mathrm{mol} / \mathrm{L}$ or creatinine clearance $<60 \mathrm{ml} / \mathrm{min}$. Statistical indicators included sex, age of onset, duration of disease, blood pressure, proteinuria, hematuria, serum IgA, renal function, serum uric acid, blood cholesterol, triglycerides, prodromal infection and so on [2].

The average number of glomeruli in renal biopsy samples was $(20.7 \pm 10.0)$. The renal biopsy specimens were divided into three groups and examined by optical microscopy (including HE, PAS, Masson and PASM staining), immunofluorescence (including Ig A, Ig G, Ig M, C3, Clq, fibrin-associated antigen) and electron microscopy. According to Lee's grading criteria the pathological changes in the kidneys were divided into 5. Glomerular, tubulointerstitial and vascular disease pathological parameters, the use of Katafuchi semi-quantitative standard; glomerular lesion score of 0 to 12 points, including mesangial cells and mesangial matrix hyperplasia ( $0 \sim 4$ points); segmental glomerular lesions such as crescent formation, adhesion with the balloon wall, segmental sclerosis and segmental capillary wall fibrosis (0-4); glomerulus Sexual sclerosis (0-4 points). 
Points for the assessment criteria: (1) no, 0 points; (2) <25\%, 1 point; (3) 25\% 50\%, 2 points; (4) 51\% 75\%, 3; (5) > 75\%, 4 points. Tubulointerstitial scores ranged from 0 to 9 and included interstitial inflammatory cell infiltrates (0-3), interstitial fibrosis (0-3), and tubular atrophy (0-3). Vascular score 0 to 6, including thickening of the vessel wall (0 to 3 points) and degeneration of the glass (0 to 3 points), the integral evaluation criteria: (1) no, 0; (2) <25\%, 1; (3) 25\% 50\%, 2 points; (4) $>50 \%$, 3 points.

\section{Findings}

The group of 412 patients with a small amount of albuminuria Ig AN patients, accounting for $10.2 \%$ of the same period renal biopsy cases, male to female ratio was $1.05: 1$, the average age of onset (28.4 \pm 8.3$)$ years. Hematuria and proteinuria were found in $90.3 \%$ (412 cases) of patients, 9.7\% (40 cases) were simple proteinuria, and 41.8\% (172 cases) had episodic gross hematuria. Among them, 112 (65.1\% the most common respiratory infections, accounting for 69.6\%, gastrointestinal infections accounted for $28.4 \%$, 2.0\% of other infections; hypertensive patients a total of 66 cases (16.0\%); 53 cases of renal dysfunction (12.9\%). Table 1 shows, normal renal function group and renal dysfunction group comparison, the age of onset and the course of renal biopsy showed no significant difference. In gender, there were significantly more men than women in the group with abnormal renal function $(\mathrm{P}<0.05)$. Occurrence of gross hematuria in the normal renal function group was significantly more than renal dysfunction group $(\mathrm{P}<0.05)$. The prevalence of hypertension in the normal renal function group was significantly lower than that in the renal dysfunction group $(10.9 \%, 50.9 \%, \mathrm{P}<0.01)$. Serum cholesterol, triglycerides and serum IgA in two groups had no significant difference. Urine protein and uric acid in renal dysfunction group were significantly higher than those in normal renal function group $(\mathrm{P}<0.05, \mathrm{P}<0.01)$. Compared with normal renal function group, serum creatinine increased significantly and creatinine clearance decreased significantly in renal dysfunction group (all $\mathrm{P}<0.01$ ). Pathological type of abnormal renal function in focal proliferative lesions with ischemic injury were mainly 28 cases (52.8\%); focal hyperplasia with crescent formation in 10 cases (18.9\%), of which are crescentic crescent 6 Cases, crescent and crescent cells coexist in 4 cases; focal hyperplastic sclerosis in 8 cases (15.1\%); focal proliferative sclerosis with ischemic renal injury in 7 cases (13.2\%). There were 23 patients (43.4\%) in grade III, 29 patients in grade IV $(54.7 \%)$ and 1 patient in grade V $(1.9 \%)$ according to Lee classification. Fifty-three patients (100\%) with abnormal renal function had glomerulosclerosis in varying degrees and 25 (47.2\%) patients showed varying degrees of crescent formation Focal renal interstitial fibrosis, tubular atrophy and varying degrees of vascular disease [3].

\section{Clinical Value Discussion}

IgA is the most common primary glomerular disease, clinical manifestations, ranging from asymptomatic microscopic hematuria or proteinuria to the classic nephrotic syndrome, and even progressive decline in renal function. At present, most studies confirm that 24h urine protein quantitation> $1 \mathrm{~g}$ is an independent risk factor for the progression of IgA N. However, even IgA N patients with mild proteinuria may not have mild kidney damage, and some of them may be exacerbated or even worsen their renal function during long-term follow-up. According to our data, 53 (12.9\%) IgA patients with a small amount of albuminuria were found to have renal dysfunction. Of the 359 patients with normal renal function, 122 (34.0\%) had grade III or higher Pathological damage, suggesting that a considerable amount of a small amount of proteinuria in patients with IgA N prognosis is not optimistic.

The patients in this group were all young and middle-aged. There were no significant differences in the age and course of disease between the normal renal function group and the abnormal renal function group. The proportion of male and female in the normal renal function group was more than that in the male group with abnormal renal function. The incidence of hypertension (50.9\%) in patients with abnormal renal function was significantly higher than that in patients with normal renal function (10.9\%), which was consistent with the severe pathological changes of renal 
pathology and focal hyperplasia with ischemic renal injury. This is consistent with the previous study found that the relative deterioration of the condition, the relatively poor prognosis of hypertensive IgA N male majority consistent. Studies showed that the presence of episodes of gross hematuria in the medical history often suggested a relatively good prognosis. This study found that patients with normal renal dysfunction, episodes of gross hematuria are more common, accounting for $47.4 \%$, while renal dysfunction group episodes of gross hematuria accounted for only $3.8 \%$, two groups were statistically significant [4].

Elevated blood IgA in the normal renal function group and renal dysfunction group no significant difference. Most studies have confirmed that elevated serum uric acid is an independent risk factor for IgA $\mathrm{N}$ prognosis. In our study, serum uric acid was significantly higher in patients with abnormal renal function than in patients with normal renal function. However, decreased anomalies when the impact of the need to further study confirmed. Renal dysfunction group of urine protein was higher than the normal renal function group, two groups were statistically significant. Tip Even in $24 \mathrm{~h}$ urine protein quantitation $<1 \mathrm{~g}$, the amount of urine protein may also affect the prognosis.

IgA $\mathrm{N}$ not only diversified clinical manifestations, renal pathology also showed diversification. The study found that both the normal renal function group and renal dysfunction group can be seen in the pathological type of focal hyperplasia with some crescent formation, focal hyperplasia with ischemic renal injury and focal proliferative sclerosis. The pathological type of abnormal renal function group was focal hyperplasia with ischemic renal injury, accounting for $52.8 \%$, followed by focal hyperplasia with crescent formation, accounting for $18.9 \%$, of which $40 \%$ were crescentus and fibrosis Month body coexistence; normal renal function group with mild mesangial hyperplasia and focal hyperplasia, accounting for $86.6 \%$, followed by focal hyperplasia with crescent formation, accounting for $3.3 \%$, of which $83.3 \%$ of the presence of crescent. Tip in a small amount of proteinuria IgA $\mathrm{N}$ patients with a considerable number of patients with renal pathological active lesions, the clinical need for active treatment. In addition, the normal renal function group with focal hyperplasia with ischemic renal injury accounted for 3.1\%; focal hyperplastic necrosis accounted for 3.1\%; focal hyperplastic sclerosis accounted for $1.9 \% ; 34.0 \%$ of Lee III grade above pathological lesions; $35.9 \%$ there are different degrees $37.3 \%$ showed varying degrees of crescent formation, $27.8 \%$ had focal renal interstitial fibrosis, $49.0 \%$ had renal tubular atrophy, and $74.9 \%$ had varying degrees of vascular disease. Ig AN patients with a small amount of proteinuria with normal renal function may have severe renal pathological lesions and may present with pathological types of IgA $\mathrm{N}$ patients with abnormal renal function but are associated with proteinuria and high proteinuria in IgA $\mathrm{N}$ patients with abnormal renal function the level of blood pressure is relatively light. This part of the patient with the extension of the disease will soon develop to renal dysfunction, the need for clinical follow-up results confirmed.

This study of patients with renal biopsy pathological indicators and clinical indicators of the correlation between the analysis as follows: M0 / M1 with blood pressure, urinary protein and serum creatinine levels increased aggravating, studies have confirmed mesangial cell proliferation imbalance Is one of the important factors leading to glomerular sclerosis, leading to poor prognosis, some scholars that it can not be used as an indicator of prognosis, but it is worth noting that most patients with mesangial cell hyperplasia received sugar The treatment of corticosteroids, which may to some extent affect the prognosis of its judgments. The study failed to confirm S0 / S1 and blood pressure, urine protein is related, but with the severity of the sclerosis increased serum creatinine, consider the reason is that glomerular segmental sclerosis as a chronic injury, need to be done Long-term follow-up to further confirm the prognosis. E0 / E1 aggravates with the increase of serum creatinine and urinary protein, and gradually decreases with the increase of blood pressure. In Oxford, this pathological index is considered as the indicator with the weakest prognosis, but the glucocorticoid Hormones and immunosuppressive agents for the treatment of pathological indicators, so it does not exclude the interference of drug treatment, the study confirmed its relevance to clinical indicators, can be used as a prognostic pathological indicators [5]. 


\section{Conclusions}

Pathological type of renal biopsy patients with primary glomerular disease, the incidence of membranous nephropathy increased year by year, IgA nephropathy incidence decreased year by year, the incidence of membranous nephropathy in the past five years than IgA nephropathy [5]; lupus nephritis is The most common pathological types of secondary kidney disease; nephrotic syndrome and chronic glomerulonephritis are the most common clinical diagnoses in renal biopsy patients. Renal biopsy has ameliorating the clinical diagnosis of the disease; ultrapathological diagnosis has an important effect on the final result of renal pathology.

\section{References}

[1] Zhang L, Wang F, Wang L, et al. Prevalence of chronic kidney disease in China: a cross-sectional survey [J]. Lancet, 2012, 379 (9818):815-822.

[2] Li L. End-stage renal disease in China [J]. Kidney international, 1996, 49 (1):287-301.

[3] Simon P1, Ramee MP, Boulahrouz R, et al. Epidemiologic data of primary glomerular diseases in western France[J]. Kidney Int, 2004, 66 (3):905-908.

[4] Covic A1, Schiller A, Volovat C, et al. Epidemiology of renal disease in Romania: a 10-year review of two regional renal biopsy databases [J]. Nephrol Dial Transplant, 2006, 21 (2):419-424.

[5] Hsiao K C. Ten-Year Registry of Native Kidney Biopsy from a Single Center in Taichung [J]. Acta Nephrologica, 2012, 26: 68-73.

[6] Iversen P, Brun C. Aspiration biopsy of the kidney. Am J Med, 1951, 11 (3):324. 